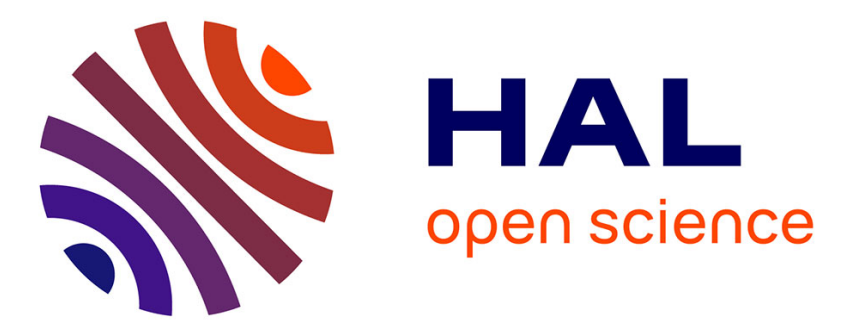

\title{
How the study of Listeria monocytogenes has led to new concepts in biology.
}

\author{
Nathalie Rolhion, Pascale Cossart
}

\section{To cite this version:}

Nathalie Rolhion, Pascale Cossart. How the study of Listeria monocytogenes has led to new concepts in biology.. Future Microbiology, 2017, 12 (7), pp.621-638. 10.2217/fmb-2016-0221 . pasteur-01574990

\section{HAL Id: pasteur-01574990}

\section{https://hal-pasteur.archives-ouvertes.fr/pasteur-01574990}

Submitted on 30 Aug 2017

HAL is a multi-disciplinary open access archive for the deposit and dissemination of scientific research documents, whether they are published or not. The documents may come from teaching and research institutions in France or abroad, or from public or private research centers.
L'archive ouverte pluridisciplinaire HAL, est destinée au dépôt et à la diffusion de documents scientifiques de niveau recherche, publiés ou non, émanant des établissements d'enseignement et de recherche français ou étrangers, des laboratoires publics ou privés.

\section{(1) $\$(0)$}

Distributed under a Creative Commons Attribution - NonCommercial - ShareAlikel 4.0 
Title

How the study of Listeria monocytogenes has led to new concepts in biology

\section{Authors}

Nathalie Rolhion and Pascale Cossart ${ }^{*}$

\section{Affiliations}

Institut Pasteur, Unité des Interactions Bactéries-Cellules, Département de Biologie Cellulaire et Infection, F-75015 Paris, France.

Inserm, U604, F-75015 Paris, France.

INRA, Unité sous-contrat 2020, F-75015 Paris, France.

* Correspondence to: pascale.cossart@pasteur.fr 


\begin{abstract}
The opportunistic intracellular bacterial pathogen Listeria monocytogenes has in 30 years emerged as an exceptional bacterial model system in infection biology. Research on this bacterium has provided considerable insight into how pathogenic bacteria adapt to mammalian hosts, invade eukaryotic cells, move intracellularly, interfere with host cell functions and disseminate within tissues. It also contributed to unveil features of normal host cells pathways and unsuspected functions of previously known cellular proteins. This review provides an updated overview of our knowledge on this pathogen. In many examples, findings on Listeria monocytogenes provided the basis for new concepts in bacterial regulation, cell biology and infection processes.
\end{abstract}

\title{
Key words
}

Listeria monocytogenes, cellular microbiology, virulence, microbiota, RNA-based regulation, PrfA, actin, post-translational modifications 


\section{Introduction}

Research on infection by pathogenic micro-organisms aims at understanding the molecular mechanisms used by pathogens to establish an infection. It often provides insights into fundamental aspects of cell biology and immunology. For example, the mechanism used by enveloped virus to enter host cells helped explaining receptor-mediated endocytosis and intracellular transport. Listeria monocytogenes, the causative agent of listeriosis, has also led to a number of discoveries in various fields that we will discuss in this review.

L. monocytogenes was identified by E.G.D. Murray in 1926 as a Gram-positive bacillus responsible for epidemic cases of mononucleosis in rabbits and guinea pigs in animal care houses in Cambridge (UK) and named as Bacillus monocytogenes [1]. A few years later, it was shown to cause sporadic cases of meningitis in humans [2] and was subsequently recognized as an opportunistic food-borne pathogen of human, cattle and wild animals [3]. This bacterium is responsible for gastroenteritis in healthy individuals, and meningitis and septicemia in immunocompromised individuals. Pregnant women, particularly those in their third trimester, can develop L. monocytogenes induced chorioamnionitis (an infection of placental tissues) and infection of the foetus leading to abortion in $20-50 \%$ of cases [4]. Cases of listeriosis are generally sporadic but epidemics occur, such as that of 2011 in USA that resulted from contaminated cantaloupes and for which 147 cases and 33 deaths have been reported [5]. The center for disease control (CDC) estimates that L. monocytogenes causes approximately 1600 human cases and 260 deaths annually in the USA (mean annual incidence 0.26 cases per 100,000 individuals, [6]). In Europe, in 2011, 1470 cases of listeriosis were reported with a mortality rate of $12.7 \%$ (mean annual incidence 0.39 cases per 100,000 residents, [7]). Early diagnosis of listeriosis and antibiotic treatment (involving both amoxicillin and gentamicin) are critical to prevent the devastating outcome and the neurological consequences of the disease. 
L. monocytogenes is widespread in the environment and is commonly found in water, soil and decaying vegetation. It is particularly well adapted to growth in high salt concentrations and/or at low temperatures, conditions normally used for food conservation and making it difficult for the food industry to rely on these conditions to control listerial contamination. Following ingestion of contaminated food (such as cheese, deli meat, vegetables, dairy products...), L. monocytogenes survives acidic conditions of the stomach and bile acids encountered within the gastrointestinal [8]. It crosses the intestinal barrier by invading the intestinal epithelium. Via the lymph and the blood, it reaches the liver and the spleen. Bacteria can then disseminate to the brain and the placenta, after crossing the bloodbrain barrier and the materno-fetal barrier, respectively. The bacterium has the remarkable ability to invade, survive and actively multiply within professional phagocytes and a number of non-phagocytic cells, to spread directly from cell-to-cell and to evade innate immunity (Figure 1). During infection, L. monocytogenes uses a series of virulence factors whose production is spatiotemporally regulated by both protein-mediated and RNA-mediated regulatory mechanisms (for reviews, $[9,10])$. These secreted or surface-exposed virulence factors allow L. monocytogenes to exploit and/or compromise host cell functions and also promote its survival.

This review highlights some of the strategies used by L. monocytogenes to subvert its host functions focusing on those in which this bacterium has opened new perspectives in bacterial gene expression regulation, cell biology, innate immunity and in vivo infection. We report how the study of virulence gene expression regulation in L. monocytogenes has led to never described mechanisms of regulation. We then discuss how bacterial infection has enabled us to explore or even reconsider cell biology and immunology processes. We highlight progress in infection biology, focusing on the effect of L. monocytogenes on gut 
microbiota. At the end of this review, we discuss future perspectives offered by the study of host-pathogen interactions.

\section{New concepts in microbial gene expression regulation}

PrfA, a tightly controlled regulator

Environmental pathogens, such as L. monocytogenes, are microorganisms that normally spend a large part of their lifecycle in the environment, and when present into human hosts can cause disease. This suggests that these pathogens must maintain a broad array of survival strategies to maintain life under these various conditions. In addition, to colonize their hosts successfully, they must regulate virulence gene expression appropriately. Coordinated virulence gene expression in L. monocytogenes is orchestrated by PrfA, a transcription factor of the cAMP receptor protein (CRP) family with a consensus binding site in the -35 region of the promoter $[11,12]$. PrfA directly regulates transcription of the major virulence factors, including the surface protein internalins InlA and InlB, the secreted protein InlC, the pore-forming toxin listeriolysin O (LLO), the phospholipases PlcA and PlcB, the metalloprotease $\mathrm{Mpl}$, the actin-polymerizing protein ActA, the hexose phosphate transporter $\mathrm{Hpt}$, and is therefore referred to as the master virulence regulator in L. monocytogenes [12, 13]. Strains lacking $\operatorname{prfA}$ are totally avirulent $[11,13]$.

Expression and activity of PrfA are tightly regulated at the transcriptional and posttranscriptional levels (Figure 2 and as discussed in the next section). Until recently, there were several lines of evidence suggesting that PrfA is also regulated at the post-translational level. Firstly, proteins of the CRP family generally require the binding of small-molecule cofactors or other forms of post-translational modification for full activity. Secondly, 
mutations within an identified PrfA cofactor-binding pocket impair PrfA activation in the cytosol and reduce bacterial virulence $[14,15]$. Finally, it was clear that PrfA is activated in the cytosol of host cells, but, even after decades of study, the biochemical mechanism by which PrfA detects the intracellular environment was not understood. Last year, by analyzing how L. monocytogenes recognizes and responds to its intracellular niche of the mammalian cell cytosol, Reniere and colleagues demonstrated that glutathione, found abundantly in the host cytoplasm, represents a critical signaling molecule activating virulence of this intracellular pathogen [16]. They showed that bacterial or host glutathione induces an allosteric change in the structure of PrfA and consequently activates expression of $L$. monocytogenes virulence factors (Figure 2). This study also suggested that L. monocytogenes uses the glutathione concentration to differentiate extracellular and intracellular environments and to regulate the switch from saprophytism to virulence. Since then, Burkolderia pseudomallei, an intracellular Gram-negative bacterium, was reported to sense the presence of reduced glutathione in the host cytosol, through the membrane-bound histidine sensor kinase VirA, leading to activation of its type VI secretion system, critical for infection in mammalian host [17]. Taken together, these two studies highlight that the same ubiquitous molecule can regulate virulence in both Gram-positive and Gram-negative pathogenic bacteria, through different sets of regulators and mechanisms. It seems that the unifying theme for L. monocytogenes and B. pseudomallei is the need to recognize the intracellular environment and to turn on their appropriate virulence genes necessary for pathogenesis in the cytosol. Given the central role glutathione plays in virulence regulation, it could represent a potential novel target for new anti-microbial drug strategies. In addition, one may speculate that other low-molecular-weight thiols (coenzyme A, mycothiol and bacillithiol) also play roles in activation of virulence gene expression in other pathogens.

Unconventional mechanisms regulating bacterial gene expression 
Successful host infection by pathogenic micro-organisms relies on the coordinated expression of many virulence factors and genes involved in the infection process. Over the last decade, much has been learned about the complexity of bacterial gene expression and the different levels of regulation. Regulatory RNAs are increasingly being recognized as important players in many physiological and adaptive responses in pathogenic bacteria [1820]. The first noncoding RNAs (ncRNAs) in L. monocytogenes were identified by coimmunoprecipitation with Hfq, a small RNA-binding protein required for the activity of small regulatory RNAs in prokaryotes [21] and by an in silico-based approaches [22]. Since then, the use of high-density tiling arrays and RNA deep sequencing provided a picture of the whole L. monocytogenes transcriptome in multiple conditions, allowing the discovery of many regulatory RNAs [23-28] and the annotation of hundreds of regulatory RNAs in $L$. monocytogenes, among which some play regulatory roles in virulence [29]. Public access and navigation through the results of these different studies are facilitated by Listeria browsers ([30] and [31] (unpublished)). 305 L. monocytogenes ncRNAs have now been reported, with 155 trans-acting sRNAs, 46 cis-regulatory RNAs and 104 antisense RNAs (asRNAs) [32]. They may play important roles in virulence gene expression. The study of some of these transcripts has revealed unconventional mechanisms of bacterial gene expression regulation and highlighted an intricate interlink between RNA-based regulatory mechanisms. These mechanisms have been recently reviewed and two reviews are highly recommended [29, 33]. Here, we will briefly illustrate that studying L. monocytogenes led to the discovery of (i) the first RNA thermosensor regulating virulence (5'-UTR of $\operatorname{prfA}$ ), (ii) the first riboswitchderived RNA able to act in trans (sreA), (iii) a riboswitch-regulated antisense RNA (aspocR), (iv) the sequestration of an antiterminator by a riboswitch-regulated ncRNA (rli55), (v) the ribo-regulation of antibiotic resistance genes, (vi) the excludon concept and (vii) an atypical CRISPR. 
RNA thermosensor. Soon after the characterization of PrfA as an activator of the expression of $h l y$, the gene encoding LLO, the expression of PrfA-dependent virulence genes was found to be thermoregulated in L. monocytogenes [34]. In addition, at low temperature, PrfA is undetectable, although prfA transcripts are present [35]. The mechanism of this regulation relies on the 5'-UTR of the prfA transcript, which is a thermosensor adopting alternative structures at different temperatures which therefore inhibit or activate PrfA translation [36] (Figure 2). Indeed, at low temperatures $\left(30^{\circ} \mathrm{C}\right)$, the $5^{\prime}$-UTR of $p r f A$ adopts a stable stem-loop structure, occluding the Shine-Dalgarno (SD) sequence and thereby preventing the binding of the $30 \mathrm{~S}$ ribosomal subunit, whereas at higher temperatures $\left(37^{\circ} \mathrm{C}\right)$, such as those encountered in warm-blood organims, the stem-loop structure opens, exposing the SD site and leading to PrfA translation. The PrfA RNA thermosensor was the first RNA thermosensor regulating virulence discovered. RNA-based thermosensors, while rare, have also been identified in other bacteria, such as Escherichia coli [37]. They control the expression of different genes, including genes involved in virulence [38]. For example, one RNA thermosensor located upstream of the virulence regulator gene lcrF of Yersinia pseudotuberculosis has been described to regulate expression of the ysc type III secretion genes and the yop effectors and to promote tissue colonization [39].

Riboswitch-derived regulatory transcript. PrfA expression is also regulated by trans-acting riboswitch-derived transcripts. Typical riboswitches are present in the 5 ' UTRs of the mRNA they control and, upon binding of a ligand (metabolite, metal ions, cyclic-diGMP...), change their conformation usually resulting in translation arrest, premature transcription arrest or both [40]. Transcripts regulated by riboswitches usually encode genes that are involved in the biosynthesis of the molecule that regulates the riboswitch. The Sadenosyl-methionine (SAM) riboswitch SreA regulates in cis expression of genes involved in methionine and cysteine metabolism, but interacts also with the 5'UTR of prfA mRNA [41] 
(Figure 2). This interaction decreases prfA translation. This is the first and so far unique example of a riboswitch derived RNA able to act in trans. In addition, this nutrient-mediated trans-regulation of $\operatorname{prfA}$ expression coupled to its temperature-mediated cis-regulation described above allows L. monocytogenes to sense its environment and regulate prfA expression accordingly. The large number of mechanisms that regulate PrfA is indicative of its importance during infection.

Riboswitch-regulated non coding RNA. Another atypical riboswitch is one dependent on vitamin $\mathrm{B}_{12}$, located at the 3' end of a gene and in antisense orientation to the downstream gene pocR and controlling the expression of an RNA antisense to pocR, aspocR [42] (Figure 3A). PocR is a transcriptional factor activating expression of genes involved in propanediol catabolism and vitamin $\mathrm{B}_{12}$ biosynthesis, both pathways being intimately linked as propanediol utilization requires vitamin $B_{12}$ as a cofactor. In absence of vitamin $B_{12}$, the riboswitch allows transcription of aspocR and thus blocks that of pocR. In presence of vitamin $\mathrm{B}_{12}$, the riboswitch arrests prematurely the transcription of aspocR, enabling that of pocR. Therefore, this mechanism ensures the transcription of pocR and thereby that of the PocR-regulon only when both propanediol and vitamin $\mathrm{B}_{12}$ are present in the growth medium.

Another L. monocytogenes vitamin $\mathrm{B}_{12}$ riboswitch is involved in regulation of the ethanolamine utilization operon (eut) [43] (Figure 3B). In the presence of ethanolamine, the eut operon is activated by the EutVW two-component system in which the sensor histidine kinase EutW phosphorylates the antiterminator EutV. However, in absence of vitamin $\mathrm{B}_{12}$, the activated EutV protein is sequestered by a ncRNA called rli55, whose expression is controlled by a vitamin $\mathrm{B}_{12}$ riboswitch. In presence of vitamin $\mathrm{B}_{12}$, the riboswitch terminates prematurely the transcription of rli55, EutV is no longer titrated and can therefore mediate antitermination, allowing expression of eut genes. Thus, upregulation of the eut operon only occurs when the two nutrients are present - the substrate ethanolamine and the co-factor 
vitamin B12. The expressions of pocR and eut operon are both regulated by vitamin B12 riboswitches and antisense RNA or an anti-terminator, respectively. This multiple layer of regulation indicates a complex and tightly regulated interlink between RNA-based regulators in L. monocytogenes. Such complex and layered RNA-based regulation of utilization of ethanolamine has also been reported in the pathogen Enterococcus faecalis [44].

Riboswitches regulating antibiotic resistance genes. The vast majority of antibiotics affecting translation have RNA binding properties and it has been speculated that RNA-based regulation could be involved in antibiotic resistance genes expression. Recently, several antibiotics resistance genes regulated via termination-based ribo-regulators were identified in L. monocytogenes [24]. Indeed, the discovery of ribo-regulators that specifically respond to antibiotics occurred by applying term-seq, a method enabling quantitative mapping of all exposed RNA 3' ends, to L. monocytogenes bacteria grown in the presence or absence of antibiotic, such as lincomycin. This method was also performed on Bacillus subtilis, E. faecalis and human oral microbiota and allowed the identification of many genes ribo-regulated by antibiotics, illustrating that conditional transcription represents a general feature for antibiotic resistance. The discovery of such new regulatory mechanisms controlling antibiotic resistance genes has provided a new window on antibiotic resistance expression, suggesting that these ribo-regulators might be excellent drug targets.

Excludon. High-resolution transcriptome of L. monocytogenes highlighted a class of very long asRNAs (lasRNAs) which display a dual function as both asRNA and mRNA and encoded by an "excludon" [27, 28]. An excludon typically consists of two divergent transcriptional units overlapped by a long asRNA that inhibits the expression of one of the two divergent operons, but whose distal part harbors information as an mRNA, activating therefore the expression of the other operon (Figure 3C). The first excludon discovered was the $\operatorname{mog} R$ locus in L. monogytogenes and excludons are conserved in other Listeria species 
and also in a more distantly related bacterium Clostridium difficile [28]. It is highly possible that excludons exist not only in bacteria, but also in other organisms.

CRISPR. A class of ncRNAs, named CRISPRs (clustered regularly interspaced short palindromic repeats) mediates prokaryotic adaptive immunity against invading bacteriophages and conjugative plasmids [45]. CRISPR RNAs processing and function rely on nucleases or helicases encoded by CRISPR-associated (cas) genes. Unlike other CRISPRs, L. monocytogenes rliB is an atypical member of the CRISPR family, devoided of cas genes [46]. Biochemistry experiments showed that it is a substrate for polynucleotide phosphorylase (PNPase), an enzyme which acts as an exoribonuclease of single stranded target RNAs and that PNPase is required for the rliB-CRISPR mediated DNA interference. Therefore, characterization of the rliB-CRISPR shed light on a new and unexpected function for PNPase in CRISPR system.

\section{Concepts in cell biology derived from the analysis of $L$. monocytogenes infections}

L. monocytogenes is able to invade a number of non-phagocytic cells. This entry is induced by interactions between bacterial surface proteins, InlA and InlB, and their respective receptors E-cadherin (E-Cad) and Met expressed at the host cell surface. These interactions trigger cell-signalling cascades, leading to actin polymerisation and host cell membrane remodelling, which in turn allow listerial engulfment via a zipper mechanism and formation of an internalisation vacuole. After escape from the vacuole, bacteria multiply and move in the cytosol, via recruitment of the actin-nucleating complex Arp2/3 by the surface-exposed bacterial protein ActA and polymerisation of an actin filaments network at one pole of the bacterium. These networks, called actin comet tails, propel the bacterium across the cytoplasm and eventually into neighbouring cells. The efficient spreading between epithelial 
cells requires the interaction of the secreted virulence protein InlC and the mammalian adaptor protein Tuba [47]. In the infected cell, L. monocytogenes impairs organelles function and target post-translational modifications at various stages of the infection process to manipulate the cell host response. We illustrate below how the research on L. monocytogenes has greatly contributed to our understanding of the structure and dynamics of the host cell cytoskeleton and to deepen our knowledge of several other central cellular processes (Figure 4).

Discovery of the Arp2/3 complex, the first actin nucleator identified in eukaryotic cells

The study of actin-based motility is one of the best examples highlighting how the study of a bacterial-induced process can yield insight into basic cellular processes. Indeed, ActA and L. monocytogenes have been exceptionally instrumental in the discovery of Arp2/3. It was first shown that $L$. monocytogenes polymerises actin at one side of the bacterium, producing an actin tail allowing bacterial motility [48, 49]. Then, it was discovered that this polymerisation of host actin is mediated by the bacterial surface protein ActA [50, 51], that it recruits the host Arp2/3 complex and that it mimics the host cell WASP proteins [52]. The Arp2/3 complex consists of seven subunits and has been classically considered as a single molecular entity since its discovery 20 years ago. However, recent studies suggest that some subunits of the complex are dispensable in some specific cellular contexts ([53-55], for review, [56]). In the case of $L$. monocytogenes infection, it was for example shown that the ARPC5 subunit is neither required for L. monocytogenes entry into host cells nor for actin tail formation and that the ARPC4 subunit is required for actin tail formation, probably at initial stages, but not for cell invasion [55]. These findings therefore highlight a previously unsuspected versatility in Arp2/3 complex composition and function [56]. 
L. monocytogenes is not the only microorganism that exploits actin for intracellular movement. (for review, [57]). Shigella flexneri recruits on its surface, via the protein IscA, NWASP which in turn activates Arp2/3 to mediate actin-based motility [58]. Rickettsia conorii and Rickettsia parkerii possess two actin-polymerizing proteins: RickA and Sca2. RickA activates the Arp $2 / 3$ complex and is involved in early stages of bacterial intracellular motility, while Sca2 is responsible for the late actin-based motility via a mechanism independent of the Arp2/3 complex [59, 60]. Mycobacterium marinum, virulent Burkolderia and vaccinia virus also move via an actin-based motility requiring Arp2/3 functions [61-65].

A role for clathrin and two myosins in bacterial entry

Investigations on InlA and InlB-mediated entries have clearly shown that these two bacterial proteins are essential for L. monocytogenes entry into non-phagocytic mammalian cells (for review, [66]). An important finding concerning the mechanisms underlying $L$. monocytogenes entry into host cells has been the discovery that the clathrin-dependent endocytic machinery is crucial in actin polymerization and therefore bacterial entry [67]. Indeed, RNA interference of major components of the endocytic machinery (e.g. clathrin, dynamin and cortactin) inhibited L. monocytogenes entry. It had before been thought that clathrin was only involved in endocytosis of small molecules (nutrients and signalling molecules) or relatively small objects $(<150 \mathrm{~nm})$ [68]. Therefore, the fact that the endocytic machinery is involved in bacterial internalization indicated that clathrin can internalize objects much larger than previously appreciated [67, 69]. Clathrin also mediates entry of large viruses and other bacteria using zipper mechanism [70-72] and also pedestal formation by the extracellular enteropathogenic E. coli (EPEC) [72]. Interestingly, by analysing the molecular events during early stages of L. monocytogenes and EPEC infection, it was 
observed that Myosin VI, a myosin that moves on actin filaments towards the minus ends, accumulates at the L. monocytogenes entry sites but not at the EPEC-induced actin-based pedestals [73]. Knowckdown of Myosin IV inhibits L. monocytogenes entry but does not reduce the recruitment of the clathrin-actin machinery at bacterial adhesion sites, suggesting the Myosin VI is the last component of this machinery and provides the pulling force for bacterial internalisation. In addition, by investigating whether the clathrin/actin interactions may also be involved in E-Cadherin-mediated cell-cell adhesion, it was discovered that clathrin is involved in the formation of adherens junctions between epithelial cells by reorganisation of actin [74]. Altogether these findings revealed a novel role for clathrin as an essential promoter of actin polymerisation and rearrangement, and reinforced the view that unrevealing pathogen infection strategies can reveal unappreciated features of normal host cells pathways.

Another unconventional myosin, the myosin VIIa, together with its ligand vezatin, are recruited at adherens junctions and at the entry site of $L$. monocytogenes, localize with actin and are required for L. monocytogenes entry [75]. To our knowledge, L. monocytogenes is the only bacterium described so far to require two unconventional myosins for its internalization.

\section{A role of septins in actin cytoskeleton rearrangements}

Septins constitute a family of 13 different GTP-binding proteins in humans involved in the regulation of cytokinesis, neurogenesis, membrane remodelling and cytoskeleton dynamics. They assemble into hetero-oligomeric complexes, form nonpolar filaments, bundles or rings, and associate with cellular membranes, actin filaments and microtubules. They are now considered as the fourth component of the cytoskeleton together with actin, microtubules and intermediate filaments [76]. SEPT9 has been found to associate with the $L$. 
monocytogenes phagosome [77]. SEPT9, and its interacting partners SEPT2 and SEPT11, are recruited at the site of entry of $L$. monocytogenes and of the pathogenic bacteria $S$. flexneri $[78,79]$. When cells are treated with cytochalasin $\mathrm{D}$, an actin polymerisation inhibitor, septin recruitment is impaired indicating that actin polymerisation happens before septin assembly [79, 80]. Surprisingly, SEPT2 contributes to bacterial invasion, while SEPT11 limits it, suggesting that different septins may play different roles in the tuning of actin-based molecular events [78, 79].

In addition to be recruited at the site of bacterial entry, septin rings assemble around both L. monocytogenes and S. flexneri actin comet tails, suggesting that septin recruitment is a general pattern wherever actin polymerisation is taking place [81]. Septins can also entrap Shigella into cage-like structures that restrict cell-to-cell spread and therefore dissemination of the bacteria [81]. In the case of L. monocytogenes, no efficient septin caging has been observed, but the above-reported studies indicate that septins play a role as scaffold for protein recruitment in several biological processes, including host-pathogens interactions.

\section{Interaction with mitochondria}

Bacterial pathogens have evolved mechanisms to target eukaryotic organelles such as the nucleus, the mitochondria or the ER-Golgi system (for review, [82]). Although $L$. monocytogenes does not invade these host cell organelles, it has developed several strategies to interfere with their functions, i.e. by inhibiting phagosomal killing and lysosomal function, by activating the ER stress response, by modulating mitochondrial dynamics, by modulating host gene expression via interference with chromatin-based regulation (for review, [83]). 
Research on L. monocytogenes has contributed to the identification of a novel mechanism of mitochondrial fission. Mitochondria are dynamics organelles that constantly undergo fusion and fission to regulate their size and subcellular distribution, and their function is tightly related to their morphology. They act as central hubs for energy production, apoptosis regulation, calcium homeostasis, biosynthesis of amino acids, lipids and nucleotides and innate immune signaling. Infection with L. monocytogenes transiently causes mitochondrial network fragmentation via the secreted pore-forming toxin LLO [84]. However, LLO-induced mitochondrial fragmentation does not follow canonical pathways. It is independent of the key fission component Drp1, revealing a novel and unique fission mechanism Drp1-independent [85]. On the other hand, it was shown that ER and actin play an active role in regulating LLO-induced mitochondria fission. In parallel to this latter study, a novel link between mitochondria and septins has been described. Indeed, SEPT2 directly interacts with Drp1 and is required for efficient localization of Drp1 at mitochondria, thus introducing septins as new players in mitochondrial dynamics [86]. In addition, mitochondria are required for an efficient assembly of septin cages to entrap S. flexneri and restrict their dissemination, identifying a new role for mitochondria in host defence [87].

Mitochondrial dynamics are also targeted by Vibrio cholerae, via a type III secretion system effector protein, VopE, which binds to mitochondrial Rho GTPases involved in mitochondria movement [88]. Other mitochondrial functions, such as apoptosis, protein trafficking, coordination of immune signaling, are also targeted by other pathogenic bacteria (for review, [89]).

Interaction with lysosomes 
Lysosomes are acidic organelles containing hydrolytic enzymes such as cathepsins and other proteases that are specialized in the degradation and recycling of macromolecules. They are able to fuse and degrade the content of a wide range of vesicles, including phagosome containing bacteria. Different stress conditions may alter the integrity of lysosomal membranes (a process known as lysosomal membrane permeabilisation (LMP)), resulting in the leakage of lysosomal content to the cytosol. It was recently shown that the secreted pore-forming toxin LLO induces LMP and the release of cathepsins in the host cytosol via its pore-forming activity [90]. Other cholesterol-dependent cytolysins, such as perfringolysin $\mathrm{O}$ and pneumolysin, secreted by other extracellular pathogenic bacteria, also induce LMP [90]. These findings unveil a novel activity of bacterial cholesterol-dependent cytolysins that may interfere with important host cellular pathways linked to lysosomal functions. So far, only few pathogenic bacteria were reported to target lysosomes by mechanisms differing from the one observed with L. monocytogenes [91-95].

Interaction with nuclear components: epigenetics and Listeria monocytogenes infection

To persist and replicate in host cells, pathogenic bacteria, such as L. monocytogenes, can directly modulate cellular signaling pathways activated upon bacterial entry and/or recognition of bacterial PAMP (Pathogen-Associated Molecular Patterns) by a variety of host receptors. In addition to the interference with transcriptional signaling cascades, the concept emerged that pathogenic bacteria can manipulate host cell transcription activity by directly acting at the nuclear level by interfering with chromatin-based regulation [96, 97]. In eukaryotic cells, DNA is packaged with histones into chromatin, a dynamic structure that maintains the stability and accessibility of the DNA genome. In addition to uncover posttranslational modifications of histones induced by L. monocytogenes infection, research 
aiming at exploring the effect of L. monocytogenes on chromatin-based regulation helped to characterize better host cells components such as SIRT2 and BAHD1.

L. monocytogenes was one of the first reported examples of an intracellular bacterium having an effect on histone modifications in host cells [98]. The L. monocytogenes poreforming toxin LLO triggers dephosphorylation of histone $\mathrm{H} 3$ on serine 10 and deacetylation of histone H4 [98]. In addition, upon binding to its receptor Met, internalin InlB induces the translocation of the host histone deacetylase sirtuin 2 (SIRT2) from the cytoplasm to the chromatin, where it deacetylates histone $\mathrm{H} 3$ on lysine 18 , leading to modifications of the host cell transcriptome [99]. So far, SIRT2 was considered to only regulate in the cytoplasm microtubule dynamics through deacetylation of alpha-tubulin and NF-kB gene expression through deacetylation of p65 and also controls adipocyte differenciation and autophagy through deacetylation FOXO1 (for review, [100]). SIRT2 is also present in the nucleus in the G2/M cell cycle transition and targets nuclear proteins such as p53. In addition, Sirt $2^{-/-}$mice can control listeriosis more efficiently than wild-type mice [99]. Therefore, the study of $L$. monocytogenes unveiled an additional nuclear function for SIRT2 in deacetylating histone H3 specifically on lysine 18 , a process which is actively exploited by the bacteria to promote its survival. Mycobacterium tuberculosis, Helicobacter pylori and other bacteria also modulate immune responses by epigenetic mechanisms, such as DNA methylation or histone modifications [96].

L. monocytogenes secretes into the cell cytosol LntA, a small protein belonging to the emerging class of nucleomodulins $[101,102]$. It was shown that LntA accumulates in the host cell nucleus and interacts directly with the previously unknown chromatin-associated repressor BAHD1, releasing it from its specifically targeted promoters and therefore allowing transcription of targeted genes $[102,103]$. In parallel to the study of the listerial effector LntA, the role of BAHD1 has been characterized in non-infected cells. This protein acts as a 
silencer by recruiting at specific promoters a set of proteins that coordinate heterochromatin assembly [104]. Therefore, L. monocytogenes has helped identifying and characterizing BAHD1.

Other nucleomodulins targeting chromatin such as the Chlamydia trachomatis histone-methyltransferase NUE, the S. flexneri phosphothreonine lyase OspF and the Shigella flexneri ubiquitin ligase IpaH9.8 have also been reported to deregulate host cell functions (for reviews $[96,101])$.

Role of SUMO in bacterial infections

L. monocytogenes was the first example of a bacterium able to dampen the host response by interfering with the host SUMOylation machinery [105]. It was shown that the LLO toxin triggers the degradation of Ubc9, an essential enzyme of the SUMOylation machinery, leading to a global decrease in the level of host SUMOylated proteins. As hyperSUMOylation impaired infection efficiency, it was suggested that deSUMOylation induced by LLO is critical to promote $L$. monocytogenes infection. Recently there have been a growing number of examples involving SUMOylation in the host-pathogen cross talk [106109].

A method combining SILAC-based quantitative proteomics and immunocapture of SUMO-modified peptides was recently developed to identify SUMOylation sites and to compare the SUMOylome of two different cell populations [110]. This method was used to characterize proteins deSUMOylated upon LLO treatment and identified DNA-binding proteins and transcription factors as candidate host factors for which SUMO-regulation may play an important role during L. monocytogenes infection. This study may also lead in a near 
future to the characterization of the role of SUMO in the function of these proteins in noninfected conditions.

\section{Role of ISG15 in bacterial infection}

Recently, the role for ISG15, an interferon-stimulated di-ubiquitin-like protein, known as an antiviral protein, was studied during L. monocytogenes infection [111]. ISG15 expression is induced by L. monocytogenes in vitro in epithelial cells as well as in vivo in mouse liver three days post-intravenous infection. ISG15 ${ }^{-/}$MEFs (Mouse Embryo Fibroblasts) and ISG15-deficient mice are more susceptible to L. monocytogenes. In addition, infection leads to ISGylation of ER and Golgi proteins and increases secretion of interleukines Il-6 and Il-8. These findings indicate that IGS15 and ISGylation counteract $L$. monocytogenes infection and reveal a new role of ISG15 as a modulator of canonical secretion.

Listeria monocytogenes and immunology research

As mentioned above L. monocytogenes has led to the discovery of new concepts in innate immunity. In addition, L. monocytogenes is used as a tool in all major immunology papers since decades. Indeed, in 1962, Mackaness showed that L. monocytogenes can resist killing by macrophages in a mouse model and that a primary infection by L. monocytogenes induces a protective cellular immune response and protection against a secondary infection [112]. Further studies then showed that the clearance of the bacteria is T-cell mediated [113]. Since then, L. monocytogenes has become a useful model for evaluation of the cellular 
interactions that are crucial for the induction and establishment of the host T-cell response. This topic has been deeply reviewed [114-116].

\section{Animal models of listeriosis}

Mouse models have been widely used for many decades and most of the currently available findings on pathophysiology and immunology of L. monocytogenes infections has been generated in mice. However, in mouse, E-cad presents a single amino-acid difference compared to human E-cad, the receptor for InlA [117]. This mutation on the $16^{\text {th }}$ amino acid of mouse E-cad prevents it from binding InlA, thereby explaining the inadequacy of the mouse model for studying the pathophysiology of listeriosis after oral inoculation, the normal route of infection. To confer the mouse permissiveness to the InlA-E-cad interaction, mouse lines expressing human E-cad have been engineered and have been instrumental to show the essential role of InlA-E-cad interaction for crossing of the intestinal barrier [118, 119]. On the other hand, a murinized InlA, able to recognize the murine receptor E-Cad, was created by substituting two amino acids (S192N and Y369N) [120]. However, this murinized InlA mediates not only E-Cad dependent internalization but also $\mathrm{N}$-Cadherin dependent internalization leading to enhanced innate immune responses and intestinal barrier damage [121].

A variety of other species such as gerbils, guinea pigs, zebrafishes, wax moths, chicken embryos, non-human primates have also occasionally been used (for reviews, [9, $122,123])$. However, it is extremely difficult to have an optimal animal model that mimics perfectly the infection process in humans, especially knowing that listeriosis appears to be an infection of immuno-compromised individuals. 


\section{Microbiota and L. monocytogenes infection}

With the recent appreciation of the important roles played by the intestinal microbiota in health and diseases, a number of studies have highlighted its specific role in protection against pathogen infection. However, many enteric pathogens have developed strategies to outcompete the intestinal community, leading to successful infection [124, 125]. In the case of L. monocytogenes infection, it was shown that the intestinal microbiota provides protection against oral infection, as germfree mice are more susceptible to L. monocytogenes infection than conventional mice [126]. Thorough transcriptional analysis of the host intestine of uninfected conventional and germfree mice and of L. monocytogenes-infected conventional and germfree mice indicated for the first time that host microRNAs expression triggered by L. monocytogenes infection is downregulated by intestinal microbiota, highlighting interplays between the pathogenic bacteria, the intestinal microbiota and the host. MicroRNAs are short ncRNAs that regulate eukaryotic gene expression by base pairing to target mRNAs and then inhibiting their translation. Modulation of host miRNAs is one of the various strategies used by bacterial pathogens to counteract host cell defenses (for review, [127]). The intestinal miRNA signature upon L. monocytogenes infection led to the establishment a list of predicted mRNA targets whose expression was affected by L. monocytogenes and the intestine microbiota [126]. Further investigations of the function of these miRNAs modulated by microbiota and/or by L. monocytogenes infection and of their putative mRNA targets will most likely provide new insights into gut homeostasis and also the impact of the host intestinal microbiota upon bacterial infection.

Some L. monocytogenes strains were recently shown to secrete listeriolysin S (LLS), a virulence factor only present in a subset of epidemic strains and specifically expressed in the intestine of orally infected mice $[128,129]$. LLS is a bacteriocin altering the host intestinal microbiota and favoring an increase in L. monocytogenes persistence. Indeed, a strain 
lacking LLS encoding gene displayed reduced bacterial loads in the intestine. In addition, analyzing the microbial community composition in the intestine indicated that LLS expression led to a decrease in Allobacullum and Alloprevotella genera, known to produce butyrate and acetic acid, respectively which inhibit either L. monocytogenes virulence gene expression or its growth. So far, bacteriocins were reported to be produced in vivo by commensal bacteria or by probiotics and to selectively inhibit the growth of competing bacteria, such as pathogens $[125,130,131]$. As an example, the commensal bacterium Bacteroides thuringiensis secretes a bacteriocin (thuricin CD) that targets Clostridia, including the pathogen $C$. difficile [132]. Therefore, LLS was the first bacteriocin described in L. monocytogenes to outcompete the mouse intestinal microbiota, leading to a successful infection. It is very likely that other pathogenic bacteria produce also bacteriocins and their study might reveal new mechanisms of pathogenicity and will help to decipher the complex relationship between the microbiota and pathogens.

\section{Conclusions and future perspectives}

Since the original paper of its discovery published ninety years ago [1], $L$. monocytogenes has become one of the most documented bacteria and listeriosis one of the most studied infectious diseases. Recent achievements were presented recently at the XIX ${ }^{\text {th }}$ ISOPOL meeting (International Symposium on Problems of Listeria and Listeriosis) and clearly illustrate the complexity of L. monocytogenes physiology and the infectious process [133]. The present review has highlighted the many facets opened following studies of $L$. monocytogenes and listeriosis. Important progress to come should arise from studies of $L$. monocytogenes interacting with the gut microbiota, from studies examining the differences 
among the various strains circulating in the clinic and in the environment and from in vivo imaging of the various cells, tissues, organs affected by the infection.

\section{Executive summary}

- L. monocytogenes is an intracellular pathogenic bacterium whose virulence factors expression is controlled by multiple RNA-mediated regulatory mechanisms (thermosensor, atypical riboswitches, excludon, CRISPR)

- Glutathione is used by L. monocytogenes as an specific indicator of intracellular environment and activates the activity of PrfA, the master virulence regulator, leading to expression of virulence factors

- L. monocytogenes manipulates several actin-related proteins (Arp2/3 complex, clathrin, unconventional myosins, septins) to promote its entry and/or its propagation

- L. monocytogenes alters mitochondria dynamics and lysosome function by novel mechanisms

- Research on L. monocytogenes broadly contributed to the emerging field named pathoepigenetics

- L. monocytogenes disturbs SUMOylome and ISGylome of infected cells

- L. monocytogenes infection occurs in the context of a complex microbiota, and understanding the interplays between the pathogenic bacteria, the intestinal microbiota and the host will be a major challenge. 


\section{References}

1. Murray EGDW, R.A. Swann, M.B.R. . A disease of rabbits characterised by a large mononuclear leucocytosis, caused by a hitherto undescribed bacillus Bacterium monocytogenes J. Pathol 29 407-439 (1926).

2. Burn CG. Characteristics of a new species of the genus Listerella obtained from human sources. J. Bacteriol 30 573-591 (1935).

3. Gray ML, Killinger AH. Listeria monocytogenes and listeric infections. Bacteriol Rev 30(2), 309-382 (1966).

4. Lamont RF, Sobel J, Mazaki-Tovi S et al. Listeriosis in human pregnancy: a systematic review. J Perinat Med 39(3), 227-236 (2011).

5. http://www.cdc.gov/listeria/outbreaks/cantaloupes-jensen-farms.

6. http://www.cdc.gov/listeria/statistics.html.

7. https://www.efsa.europa.eu/fr/topics/topic/listeria.

8. Gahan CG, Hill C. Gastrointestinal phase of Listeria monocytogenes infection. J Appl Microbiol 98(6), 1345-1353 (2005).

9. Cossart P. Illuminating the landscape of host-pathogen interactions with the bacterium Listeria monocytogenes. Proc Natl Acad Sci U S A 108(49), 19484-19491 (2011).

10. Cossart P, Lebreton A. A trip in the "New Microbiology" with the bacterial pathogen Listeria monocytogenes. FEBS Lett 588(15), 2437-2445 (2014).

11. Chakraborty $\mathrm{T}$, Leimeister-Wachter $\mathrm{M}$, Domann $\mathrm{E}$ et al. Coordinate regulation of virulence genes in Listeria monocytogenes requires the product of the prfA gene. J Bacteriol 174(2), 568-574 (1992).

12. Freitag NE, Port GC, Miner MD. Listeria monocytogenes - from saprophyte to intracellular pathogen. Nat Rev Microbiol 7(9), 623-628 (2009).

13. Xayarath B, Freitag NE. Optimizing the balance between host and environmental survival skills: lessons learned from Listeria monocytogenes. Future Microbiol 7(7), 839-852 (2012).

14. Eiting M, Hageluken G, Schubert WD, Heinz DW. The mutation G145S in PrfA, a key virulence regulator of Listeria monocytogenes, increases DNA-binding affinity by stabilizing the HTH motif. Mol Microbiol 56(2), 433-446 (2005).

15. Xayarath B, Volz KW, Smart JI, Freitag NE. Probing the role of protein surface charge in the activation of PrfA, the central regulator of Listeria monocytogenes pathogenesis. PLoS One 6(8), e23502 (2011).

16. Reniere ML, Whiteley AT, Hamilton KL et al. Glutathione activates virulence gene expression of an intracellular pathogen. Nature 517(7533), 170-173 (2015).

17. Wong J, Chen Y, Gan YH. Host Cytosolic Glutathione Sensing by a Membrane Histidine Kinase Activates the Type VI Secretion System in an Intracellular Bacterium. Cell Host Microbe 18(1), 38-48 (2015).

18. Caldelari I, Chao Y, Romby P, Vogel J. RNA-mediated regulation in pathogenic bacteria. Cold Spring Harb Perspect Med 3(9), a010298 (2013).

19. Gripenland J, Netterling S, Loh E, Tiensuu T, Toledo-Arana A, Johansson J. RNAs: regulators of bacterial virulence. Nat Rev Microbiol 8(12), 857-866 (2010).

20. Storz G, Vogel J, Wassarman KM. Regulation by small RNAs in bacteria: expanding frontiers. Mol Cell 43(6), 880-891 (2011).

21. Christiansen JK, Nielsen JS, Ebersbach T, Valentin-Hansen P, Sogaard-Andersen L, Kallipolitis $\mathrm{BH}$. Identification of small Hfq-binding RNAs in Listeria monocytogenes. RNA 12(7), 13831396 (2006).

22. Mandin P, Repoila F, Vergassola M, Geissmann T, Cossart P. Identification of new noncoding RNAs in Listeria monocytogenes and prediction of mRNA targets. Nucleic Acids Res 35(3), 962-974 (2007). 
23. Behrens $\mathrm{S}$, Widder $\mathrm{S}$, Mannala GK et al. Ultra deep sequencing of Listeria monocytogenes sRNA transcriptome revealed new antisense RNAs. PLoS One 9(2), e83979 (2014).

24. Dar D, Shamir M, Mellin JR et al. Term-seq reveals abundant ribo-regulation of antibiotics resistance in bacteria. Science 352(6282), aad9822 (2016).

25. Mraheil MA, Billion A, Mohamed W et al. The intracellular sRNA transcriptome of Listeria monocytogenes during growth in macrophages. Nucleic Acids Res 39(10), 4235-4248 (2011).

26. Oliver HF, Orsi RH, Ponnala L et al. Deep RNA sequencing of L. monocytogenes reveals overlapping and extensive stationary phase and sigma B-dependent transcriptomes, including multiple highly transcribed noncoding RNAs. BMC Genomics 10641 (2009).

27. Toledo-Arana A, Dussurget $\mathrm{O}$, Nikitas $\mathrm{G}$ et al. The Listeria transcriptional landscape from saprophytism to virulence. Nature 459(7249), 950-956 (2009).

28. Wurtzel O, Sesto N, Mellin JR et al. Comparative transcriptomics of pathogenic and nonpathogenic Listeria species. Mol Syst Biol 8583 (2012).

29. Sesto N, Koutero M, Cossart P. Bacterial and cellular RNAs at work during Listeria infection. Future Microbiol 9(9), 1025-1037 (2014).

30. http://www.weizmann.ac.il/molgen/Sorek/listeria_browser/.

31. http://listeriomics.pasteur.fr.

32. Becavin $\mathrm{C}$, Bouchier $\mathrm{C}$, Lechat $\mathrm{P}$ et al. Comparison of widely used Listeria monocytogenes strains EGD, 10403S, and EGD-e highlights genomic variations underlying differences in pathogenicity. MBio 5(2), e00969-00914 (2014).

33. Lebreton A, Cossart P. RNA- and protein-mediated control of Listeria monocytogenes virulence gene expression. RNA Biol doi:10.1080/15476286.2016.1189069 1-11 (2016).

34. Leimeister-Wachter M, Domann E, Chakraborty T. The expression of virulence genes in Listeria monocytogenes is thermoregulated. J Bacteriol 174(3), 947-952 (1992).

35. Renzoni A, Klarsfeld A, Dramsi S, Cossart P. Evidence that PrfA, the pleiotropic activator of virulence genes in Listeria monocytogenes, can be present but inactive. Infect Immun 65(4), 1515-1518 (1997).

36. Johansson J, Mandin P, Renzoni A, Chiaruttini C, Springer M, Cossart P. An RNA thermosensor controls expression of virulence genes in Listeria monocytogenes. Cell 110(5), 551-561 (2002).

37. Giuliodori AM, Di Pietro F, Marzi $S$ et al. The cspA mRNA is a thermosensor that modulates translation of the cold-shock protein CspA. Mol Cell 37(1), 21-33 (2010).

38. Johansson J. RNA thermosensors in bacterial pathogens. Contrib Microbiol 16 150-160 (2009).

39. Bohme K, Steinmann R, Kortmann J et al. Concerted actions of a thermo-labile regulator and a unique intergenic RNA thermosensor control Yersinia virulence. PLoS Pathog 8(2), e1002518 (2012).

40. Serganov A, Nudler E. A decade of riboswitches. Cell 152(1-2), 17-24 (2013).

41. Loh E, Dussurget $\mathrm{O}$, Gripenland J et al. A trans-acting riboswitch controls expression of the virulence regulator PrfA in Listeria monocytogenes. Cell 139(4), 770-779 (2009).

42. Mellin JR, Tiensuu T, Becavin C, Gouin E, Johansson J, Cossart P. A riboswitch-regulated antisense RNA in Listeria monocytogenes. Proc Natl Acad Sci U S A 110(32), 13132-13137 (2013).

43. Mellin JR, Koutero M, Dar D, Nahori MA, Sorek R, Cossart P. Riboswitches. Sequestration of a two-component response regulator by a riboswitch-regulated noncoding RNA. Science 345(6199), 940-943 (2014).

44. Debroy S, Gebbie M, Ramesh A et al. Riboswitches. A riboswitch-containing sRNA controls gene expression by sequestration of a response regulator. Science 345(6199), 937-940 (2014).

45. Horvath P, Barrangou R. CRISPR/Cas, the immune system of bacteria and archaea. Science 327(5962), 167-170 (2010). 
46. Sesto N, Touchon M, Andrade JM et al. A PNPase dependent CRISPR System in Listeria. PLoS Genet 10(1), e1004065 (2014).

47. Rajabian T, Gavicherla B, Heisig M et al. The bacterial virulence factor InIC perturbs apical cell junctions and promotes cell-to-cell spread of Listeria. Nat Cell Biol 11(10), 1212-1218 (2009).

48. Dabiri GA, Sanger JM, Portnoy DA, Southwick FS. Listeria monocytogenes moves rapidly through the host-cell cytoplasm by inducing directional actin assembly. Proc Natl Acad Sci U S A 87(16), 6068-6072 (1990).

49. Tilney LG, Portnoy DA. Actin filaments and the growth, movement, and spread of the intracellular bacterial parasite, Listeria monocytogenes. J Cell Biol 109(4 Pt 1), 1597-1608 (1989).

50. Domann $\mathrm{E}$, Wehland J, Rohde $\mathrm{M}$ et al. A novel bacterial virulence gene in Listeria monocytogenes required for host cell microfilament interaction with homology to the proline-rich region of vinculin. EMBO J 11(5), 1981-1990 (1992).

51. Kocks C, Gouin E, Tabouret M, Berche P, Ohayon H, Cossart P. L. monocytogenes-induced actin assembly requires the actA gene product, a surface protein. Cell 68(3), 521-531 (1992).

52. Welch MD, Iwamatsu A, Mitchison TJ. Actin polymerization is induced by Arp $2 / 3$ protein complex at the surface of Listeria monocytogenes. Nature 385(6613), 265-269 (1997).

53. Abella JV, Galloni C, Pernier J et al. Isoform diversity in the Arp2/3 complex determines actin filament dynamics. Nat Cell Biol 18(1), 76-86 (2016).

54. Chorev DS, Moscovitz O, Geiger B, Sharon M. Regulation of focal adhesion formation by a vinculin-Arp2/3 hybrid complex. Nat Commun 53758 (2014).

55. Kuhbacher A, Emmenlauer $M$, Ramo $P$ et al. Genome-Wide siRNA Screen Identifies Complementary Signaling Pathways Involved in Listeria Infection and Reveals Different Actin Nucleation Mechanisms during Listeria Cell Invasion and Actin Comet Tail Formation. MBio 6(3), e00598-00515 (2015).

56. Pizarro-Cerda J, Chorev DS, Geiger B, Cossart P. The Diverse Family of Arp2/3 Complexes. Trends Cell Biol doi:10.1016/j.tcb.2016.08.001 (2016).

57. Cossart P. Actin-based motility of pathogens: the Arp2/3 complex is a central player. Cell Microbiol 2(3), 195-205 (2000).

58. Egile C, Loisel TP, Laurent $V$ et al. Activation of the CDC42 effector N-WASP by the Shigella flexneri IcsA protein promotes actin nucleation by Arp2/3 complex and bacterial actin-based motility. J Cell Biol 146(6), 1319-1332 (1999).

59. Gouin E, Egile $C$, Dehoux $P$ et al. The RickA protein of Rickettsia conorii activates the Arp2/3 complex. Nature 427(6973), 457-461 (2004).

60. Reed SC, Serio AW, Welch MD. Rickettsia parkeri invasion of diverse host cells involves an Arp2/3 complex, WAVE complex and Rho-family GTPase-dependent pathway. Cell Microbiol 14(4), 529-545 (2012).

61. Benanti EL, Nguyen CM, Welch MD. Virulent Burkholderia species mimic host actin polymerases to drive actin-based motility. Cell 161(2), 348-360 (2015).

62. Cudmore S, Cossart P, Griffiths G, Way M. Actin-based motility of vaccinia virus. Nature 378(6557), 636-638 (1995).

63. Sitthidet C, Stevens JM, Field TR, Layton AN, Korbsrisate S, Stevens MP. Actin-based motility of Burkholderia thailandensis requires a central acidic domain of BimA that recruits and activates the cellular Arp2/3 complex. J Bacteriol 192(19), 5249-5252 (2010).

64. Stamm LM, Morisaki JH, Gao LY et al. Mycobacterium marinum escapes from phagosomes and is propelled by actin-based motility. J Exp Med 198(9), 1361-1368 (2003).

65. Gouin E, Quereda JJ, Cossart P. Intracellular bacteria find the right motion. Cell 161(2), 199200 (2015).

66. Pizarro-Cerda J, Kuhbacher A, Cossart P. Entry of Listeria monocytogenes in mammalian epithelial cells: an updated view. Cold Spring Harb Perspect Med 2(11), (2012). 
67. Veiga E, Cossart P. Listeria hijacks the clathrin-dependent endocytic machinery to invade mammalian cells. Nat Cell Biol 7(9), 894-900 (2005).

68. Conner SD, Schmid SL. Regulated portals of entry into the cell. Nature 422(6927), 37-44 (2003).

69. Pizarro-Cerda J, Bonazzi M, Cossart P. Clathrin-mediated endocytosis: what works for small, also works for big. Bioessays 32(6), 496-504 (2010).

70. Cossart P, Helenius A. Endocytosis of viruses and bacteria. Cold Spring Harb Perspect Biol 6(8), (2014).

71. Ehrlich $\mathrm{M}$, Boll W, Van Oijen A et al. Endocytosis by random initiation and stabilization of clathrin-coated pits. Cell 118(5), 591-605 (2004).

72. Veiga $E$, Cossart $P$. The role of clathrin-dependent endocytosis in bacterial internalization. Trends Cell Biol 16(10), 499-504 (2006).

73. Bonazzi M, Vasudevan L, Mallet A et al. Clathrin phosphorylation is required for actin recruitment at sites of bacterial adhesion and internalization. J Cell Biol 195(3), 525-536 (2011).

74. Bonazzi M, Kuhbacher A, Toledo-Arana A et al. A common clathrin-mediated machinery coordinates cell-cell adhesion and bacterial internalization. Traffic 13(12), 1653-1666 (2012).

75. Sousa S, Cabanes D, El-Amraoui A, Petit C, Lecuit M, Cossart P. Unconventional myosin VIIa and vezatin, two proteins crucial for Listeria entry into epithelial cells. J Cell Sci 117(Pt 10), 2121-2130 (2004).

76. Mostowy S, Cossart P. Septins: the fourth component of the cytoskeleton. Nat Rev Mol Cell Biol 13(3), 183-194 (2012).

77. Pizarro-Cerda J, Jonquieres R, Gouin E, Vandekerckhove J, Garin J, Cossart P. Distinct protein patterns associated with Listeria monocytogenes InIA- or InlB-phagosomes. Cell Microbiol 4(2), 101-115 (2002).

78. Mostowy S, Danckaert A, Tham TN et al. Septin 11 restricts InlB-mediated invasion by Listeria. J Biol Chem 284(17), 11613-11621 (2009).

79. Mostowy S, Nam Tham T, Danckaert A et al. Septins regulate bacterial entry into host cells. PLoS One 4(1), e4196 (2009).

80. Huang YW, Yan M, Collins RF, Diciccio JE, Grinstein S, Trimble WS. Mammalian septins are required for phagosome formation. Mol Biol Cell 19(4), 1717-1726 (2008).

81. Mostowy S, Bonazzi M, Hamon MA et al. Entrapment of intracytosolic bacteria by septin cage-like structures. Cell Host Microbe 8(5), 433-444 (2010).

82. Escoll $\mathrm{P}$, Mondino S, Rolando M, Buchrieser $\mathrm{C}$. Targeting of host organelles by pathogenic bacteria: a sophisticated subversion strategy. Nat Rev Microbiol 14(1), 5-19 (2016).

83. Lebreton A, Stavru F, Cossart P. Organelle targeting during bacterial infection: insights from Listeria. Trends Cell Biol 25(6), 330-338 (2015).

84. Stavru F, Bouillaud F, Sartori A, Ricquier D, Cossart P. Listeria monocytogenes transiently alters mitochondrial dynamics during infection. Proc Natl Acad Sci U S A 108(9), 3612-3617 (2011).

85. Stavru F, Palmer AE, Wang C, Youle RJ, Cossart P. Atypical mitochondrial fission upon bacterial infection. Proc Natl Acad Sci U S A 110(40), 16003-16008 (2013).

86. Pagliuso A, Tham TN, Stevens JK et al. A role for septin 2 in Drp1-mediated mitochondrial fission. EMBO Rep 17(6), 858-873 (2016).

87. Sirianni A, Krokowski S, Lobato-Marquez D et al. Mitochondria mediate septin cage assembly to promote autophagy of Shigella. EMBO Rep 17(7), 1029-1043 (2016).

88. Suzuki M, Danilchanka O, Mekalanos JJ. Vibrio cholerae T3SS effector VopE modulates mitochondrial dynamics and innate immune signaling by targeting Miro GTPases. Cell Host Microbe 16(5), 581-591 (2014). 
89. Fielden LF, Kang Y, Newton HJ, Stojanovski D. Targeting mitochondria: how intravacuolar bacterial pathogens manipulate mitochondria. Cell Tissue Res doi:10.1007/s00441-016-2475$x$ (2016).

90. Malet JK, Cossart P, Ribet D. Alteration of epithelial cell lysosomal integrity induced by bacterial cholesterol-dependent cytolysins. Cell Microbiol doi:10.1111/cmi.12682 (2016).

91. Bewley MA, Naughton M, Preston J et al. Pneumolysin activates macrophage lysosomal membrane permeabilization and executes apoptosis by distinct mechanisms without membrane pore formation. MBio 5(5), e01710-01714 (2014).

92. Bewley MA, Pham TK, Marriott HM et al. Proteomic evaluation and validation of cathepsin D regulated proteins in macrophages exposed to Streptococcus pneumoniae. Mol Cell Proteomics 10(6), M111 008193 (2011).

93. Kennedy CL, Smith DJ, Lyras D, Chakravorty A, Rood Jl. Programmed cellular necrosis mediated by the pore-forming alpha-toxin from Clostridium septicum. PLoS Pathog 5(7), e1000516 (2009).

94. Matsuda S, Okada N, Kodama T, Honda T, lida T. A cytotoxic type III secretion effector of Vibrio parahaemolyticus targets vacuolar $\mathrm{H}+$-ATPase subunit $\mathrm{c}$ and ruptures host cell lysosomes. PLoS Pathog 8(7), e1002803 (2012).

95. Prince LR, Bianchi SM, Vaughan KM et al. Subversion of a lysosomal pathway regulating neutrophil apoptosis by a major bacterial toxin, pyocyanin. J Immunol 180(5), 3502-3511 (2008).

96. Bierne H, Hamon M, Cossart P. Epigenetics and bacterial infections. Cold Spring Harb Perspect Med 2(12), a010272 (2012).

97. Minarovits J. Microbe-induced epigenetic alterations in host cells: the coming era of pathoepigenetics of microbial infections. A review. Acta Microbiol Immunol Hung 56(1), 1-19 (2009).

98. Hamon MA, Batsche E, Regnault B et al. Histone modifications induced by a family of bacterial toxins. Proc Natl Acad Sci U S A 104(33), 13467-13472 (2007).

99. Eskandarian HA, Impens F, Nahori MA et al. A role for SIRT2-dependent histone H3K18 deacetylation in bacterial infection. Science 341(6145), 1238858 (2013).

100. Houtkooper RH, Pirinen E, Auwerx J. Sirtuins as regulators of metabolism and healthspan. Nat Rev Mol Cell Biol 13(4), 225-238 (2012).

101. Bierne $H$, Cossart $P$. When bacteria target the nucleus: the emerging family of nucleomodulins. Cell Microbiol 14(5), 622-633 (2012).

102. Lebreton $A$, Lakisic $G$, Job $V$ et al. A bacterial protein targets the BAHD1 chromatin complex to stimulate type III interferon response. Science 331(6022), 1319-1321 (2011).

103. Lebreton $A$, Job V, Ragon $M$ et al. Structural basis for the inhibition of the chromatin repressor BAHD1 by the bacterial nucleomodulin LntA. MBio 5(1), e00775-00713 (2014).

104. Bierne $\mathrm{H}$, Tham TN, Batsche $\mathrm{E}$ et al. Human BAHD1 promotes heterochromatic gene silencing. Proc Natl Acad Sci U S A 106(33), 13826-13831 (2009).

105. Ribet D, Hamon M, Gouin E et al. Listeria monocytogenes impairs SUMOylation for efficient infection. Nature 464(7292), 1192-1195 (2010).

106. Beyer AR, Truchan HK, May L, Walker NJ, Borjesson DL, Carlyon JA. The Anaplasma phagocytophilum effector AmpA hijacks host cell SUMOylation. Cell Microbiol 17(4), 504-519 (2015).

107. Dunphy PS, Luo T, Mcbride JW. Ehrlichia chaffeensis exploits host SUMOylation pathways to mediate effector-host interactions and promote intracellular survival. Infect Immun 82(10), 4154-4168 (2014).

108. Fritah S, Lhocine N, Golebiowski F et al. Sumoylation controls host anti-bacterial response to the gut invasive pathogen Shigella flexneri. EMBO Rep 15(9), 965-972 (2014). 
109. Kim JG, Stork W, Mudgett MB. Xanthomonas type III effector XopD desumoylates tomato transcription factor SIERF4 to suppress ethylene responses and promote pathogen growth. Cell Host Microbe 13(2), 143-154 (2013).

110. Impens F, Radoshevich L, Cossart P, Ribet D. Mapping of SUMO sites and analysis of SUMOylation changes induced by external stimuli. Proc Natl Acad Sci U S A 111(34), 1243212437 (2014).

111. Radoshevich L, Impens F, Ribet D et al. ISG15 counteracts Listeria monocytogenes infection. Elife 4 (2015).

112. Mackaness GB. Cellular resistance to infection. J Exp Med 116 381-406 (1962).

113. Ladel $\mathrm{CH}$, Flesch IE, Arnoldi J, Kaufmann SH. Studies with MHC-deficient knock-out mice reveal impact of both $\mathrm{MHC} \mathrm{I-} \mathrm{and} \mathrm{MHC} \mathrm{II-dependent} \mathrm{T}$ cell responses on Listeria monocytogenes infection. J Immunol 153(7), 3116-3122 (1994).

114. Khan SH, Badovinac VP. Listeria monocytogenes: a model pathogen to study antigen-specific memory CD8 T cell responses. Semin Immunopathol 37(3), 301-310 (2015).

115. Lara-Tejero M, Pamer EG. T cell responses to Listeria monocytogenes. Curr Opin Microbiol 7(1), 45-50 (2004).

116. Stavru F, Archambaud C, Cossart P. Cell biology and immunology of Listeria monocytogenes infections: novel insights. Immunol Rev 240(1), 160-184 (2011).

117. Lecuit M, Dramsi S, Gottardi C, Fedor-Chaiken M, Gumbiner B, Cossart P. A single amino acid in E-cadherin responsible for host specificity towards the human pathogen Listeria monocytogenes. EMBO J 18(14), 3956-3963 (1999).

118. Lecuit $\mathrm{M}$, Vandormael-Pournin S, Lefort J et al. A transgenic model for listeriosis: role of internalin in crossing the intestinal barrier. Science 292(5522), 1722-1725 (2001).

119. Disson O, Grayo S, Huillet E et al. Conjugated action of two species-specific invasion proteins for fetoplacental listeriosis. Nature 455(7216), 1114-1118 (2008).

120. Wollert $\mathrm{T}$, Pasche $\mathrm{B}$, Rochon $\mathrm{M}$ et al. Extending the host range of Listeria monocytogenes by rational protein design. Cell 129(5), 891-902 (2007).

121. Tsai $\mathrm{YH}$, Disson $\mathrm{O}$, Bierne $\mathrm{H}$, Lecuit $\mathrm{M}$. Murinization of internalin extends its receptor repertoire, altering Listeria monocytogenes cell tropism and host responses. PLoS Pathog 9(5), e1003381 (2013).

122. Hoelzer K, Pouillot R, Dennis S. Animal models of listeriosis: a comparative review of the current state of the art and lessons learned. Vet Res 4318 (2012).

123. Disson O, Lecuit M. In vitro and in vivo models to study human listeriosis: mind the gap. Microbes Infect 15(14-15), 971-980 (2013).

124. Baumler AJ, Sperandio V. Interactions between the microbiota and pathogenic bacteria in the gut. Nature 535(7610), 85-93 (2016).

125. Rolhion N, Chassaing B. When pathogenic bacteria meet the intestinal microbiota. Philos Trans R Soc Lond B Biol Sci 371(1707), (2016).

126. Archambaud C, Sismeiro O, Toedling $J$ et al. The intestinal microbiota interferes with the microRNA response upon oral Listeria infection. MBio 4(6), e00707-00713 (2013).

127. Das K, Garnica O, Dhandayuthapani S. Modulation of Host miRNAs by Intracellular Bacterial Pathogens. Front Cell Infect Microbiol 679 (2016).

128. Cotter PD, Draper LA, Lawton EM et al. Listeriolysin S, a novel peptide haemolysin associated with a subset of lineage I Listeria monocytogenes. PLoS Pathog 4(9), e1000144 (2008).

129. Quereda JJ, Dussurget O, Nahori MA et al. Bacteriocin from epidemic Listeria strains alters the host intestinal microbiota to favor infection. Proc Natl Acad Sci U S A 113(20), 5706-5711 (2016).

130. Cotter PD, Ross RP, Hill C. Bacteriocins - a viable alternative to antibiotics? Nat Rev Microbiol 11(2), 95-105 (2013).

131. Kommineni S, Bretl DJ, Lam $V$ et al. Bacteriocin production augments niche competition by enterococci in the mammalian gastrointestinal tract. Nature 526(7575), 719-722 (2015). 
132. Rea MC, Sit CS, Clayton E et al. Thuricin CD, a posttranslationally modified bacteriocin with a narrow spectrum of activity against Clostridium difficile. Proc Natl Acad Sci U S A 107(20), 9352-9357 (2010).

133. Lebreton AS, F.; Brisse, S.; Cossart, P. 1926-2016: 90 years of listeriology. Microbes and Infection doi:10.1016/j.micinf.2016.10.009 (2016).

Reference annotation (* of interest, ${ }^{* *}$ of considerable interest)

* Cossart PNAS 2011 Comprehenvise review of Listeria interactions with the host

** Cossart FEBS 2014 Review describing most recent achievements in the understanding of the host-bacterial crosstalk

** Reniere Nature 2015 Highlight of the role of glutathione in activating PrfA activity in host cytoplasm and therefore virulence gene expression in L. monocytogenes

** Dar Science 2016 Discovery of a broad role for conditional termination in regulating antibiotic resistance in bacteria using a high-throughput method

** Toledo Arana nature 2009 Extensive trasncriptomic analysis of L. monocytogenes grown in several different conditions

** Lebreton RNA biology 2016 Comprehensive review of regulatory RNAs in $L$. monocytogenes

** Johansson cell 2002 Discovery of the RNA thermosensor regulating the expression of the master virulence regulator, PrfA

* Loh cell 2009 Discovery of the first trans-acting riboswitch in L. monocytogenes

** Mellin Science 2014 and ** DebRoy Science 2014 both demonstrate that riboswitches regulate RNA

* Pizarro-Cerda trends in cell biology 2016 Opinion on the diversity of Arp2/3 complexes 
* Escoll nature reviews microbiology 2016 Comprehensive review of the mechanisms used by bacterial pathogens to target host organelles

* Hamon PNAS 2007 Demonstrates that L. monocytogenes induces dephosphorylation as well as deacetylation of histones

** Lebreton Science 2011 Identifies a secreted listerial virulence factor, LntA, targeting the chromatin repressor BAHD1 and therefore activating expression of interferon response genes.

* Baumler Nature 2016 and * Rolhion Philos Trans R Soc Lond B Biol Sci 2016 Two comprehensive and recent reviews on the interactions between the gut microbiota and pathogenic bacteria

\section{Financial disclosure}

The authors have no relevant affiliations or financial involvement with any organization or entity with a financial interest in or financial conflict with the subject matter or materials discussed in the manuscript apart from those disclosed. No writing assistance was utilized in the production of this manuscript.

\section{Acknowledgements}

We apologize to colleagues whose work could not been cited or discussed because of space limitations. We are grateful to Olivier Dussurget and David Ribet for critical reading of the manuscript, to Fabrizia Stavru, Julien K. Malet and Juan Jose Quereda for sharing microscopy images, to Alice Lebreton and Mikael Koutero for help with the figures and to 
Melodie Duval for useful discussion. Work in P.C. laboratory received financial support from Institut Pasteur, INSERM, INRA, National Research Agency (ANR) (ERANET Infect-ERA PROANTILIS ANR-13-IFEC-0004-02), the French Government's Investissement d'Avenir program, Laboratoire d'Excellence "Integrative Biology of Emerging Infectious Diseases" (ANR-10-LABX-62-IBEID), the European Research Council (ERC) (Advanced Grant \#233348 MODELIST, H2020-ERC-2014-ADG 670823-BacCellEpi), the Fondation le Roch les Mousquetaires and the Fondation Balzan. P.C. is a Senior International Research Scholar of the Howard Hughes Medical Institute.

\section{Figure legends}

Figure 1. Intracellular life cycle of $\mathbf{L}$. monocytogenes. Bacteria entry is mediated by the interactions of internalins InlA and InlB with their respective receptors E-cadherine and Met expressed at the surface of the host cell (1). Bacteria escape from the endocytic vacuole via the action of secreted factors, the pore forming toxin listeriolysin $O$ (LLO), two phospholipases PlcA and PlcB and the metalloprotease MpI (2). Bacteria then multiply in the cytosol and factors such as the internalin InlC, the sugar uptake system Hpt and the Oacetyltransferase OatA favor intracellular growth and immune escape (3). The surfaceexposed bacterial protein ActA induces the polymerisation of actin through the recrutment of the Arp2/3 complex (4). Polymerised-actin filaments, called actin comet tails, and the interaction of InlC with the actin-binding protein Tuba allow cell-to-cell spread of the bacteria (5). LLO, PlcA and PlcB allow the rupture of the two-membrane vacuole (6). Figure adapted from [10] and reprinted from [9] with the permission of PNAS. 
Figure 2. Multiple mechanisms of regulation of PrfA expression and activity. Posttranscriptional control of prfA expression, adapted from [10]. In the environment, at low temperatures, the 5'-UTR of prfA mRNA forms a closed stem-loop structure, masking the Shine-Dalgarno (SD) sequence and thereby preventing binding of ribosome. At $37^{\circ} \mathrm{C}$, the stem-loop structure melts, liberating the SD and allowing translation initiation. The SreA small RNA, which is the product of a SAM riboswitch, can also bind to the 5'-UTR of prfA mRNA and block its translation. Upon entry into host cells, the temperature switchs to $37^{\circ} \mathrm{C}$, prfA mRNA is translated but the produced PrfA protein stays inactive. After vacuolar escape, the gluthatione (GSH) found in the host cytoplasm, enters in bacteria, binds to PrfA, changes its structure and thereby activates transcription of PrfA-regulated genes (prg), leading to production of virulence factors.

Figure 3. RNA-mediated regulations of L. monocytogenes gene expression. A. The vitamin $\mathrm{B}_{12}$ riboswitch (RS) regulates the expression of the antisens aspocR, inspired from [29]. In absence of vitamin $\mathrm{B}_{12}$, the RS, aspocR is produced and inhibits the translation of pocR. In presence of vitamin $\mathrm{B}_{12}$, the $\mathrm{RS}$ terminates prematurely the transcription of aspocR, allowing production of PocR transcription factor, provided that propanediol is also present in the medium. B. Sequestration of EutV by a $B_{12}$ riboswitch, inspired by [33]. In presence of ethanolamine $(\mathrm{Ea})$, the antiterminator EutV is phosphorylated by EutW. In absence of vitamin $\mathrm{B}_{12}$, phosphorylated EutV is sequestred by rli55 and the eut genes are not transcribed. In presence of vitamin $\mathrm{B} 12$, the transcription of rli55 is terminated prematurely by the vitamin B12 riboswitch (RS). Free phosphorylated EutV can induce antitermination and expression of the eut genes. C. Example of an excludon, where a long antisens anti0677 overlaps with the fli operon, encoding the flagellum export apparatus, adapted from [29]. $\operatorname{mog} R$ is encompassed by 2 transcripts. The short one allows the production of $\operatorname{Mog} \mathrm{R}$, a 
transcriptional repressor of the flagellum genes. The long one also allows the production of the anti0677. Therefore, the excludon ensures that, via two mechanisms (inhibition of fli mRNAs mediated by the anti0677 and repression mediated by MogR), flagellum production is switched off.

Figure 4. Subversion of host cell functions by $L$. monocytogenes. (1) Actin, clathrin and septin (green fluorescence) are recruted at the site of entry of L. monocytogenes (red fluorescence). Reprints from [9] with the permission of PNAS. (2) L. monocytogenes (green fluorescence) forming actin comet tails (red fluorescence) in infected Jeg3 cell and spreading to neighboring cell (courtesy of JJ. Quereda). Secretion of LLO induces: (3) degradation of E2-SUMO ligase and a global desumoylation of host cell proteins, (4) lysosomal membrane permeabilisation (LMP) leading to neutralisation of lysosomes (caracterised by a decreased intensity of staining with Lysotracker, an acidophilic dye, courtesy of JK. Malet) and release of cathepsins in the host cytosol, and (5) calcium influx promoting the fragmentation of the mitochondrial network (courtesy of F. Stravru). The secreted internalin InlC interacts with IKK $\alpha$, preventing NF- $\kappa \mathrm{B}$ activation and thus dampening the innate immune response (6). $L$. monocytogenes also controls host gene transcription, adapted from [10] : A) LLO induces an efflux of cellular potassium leading to dephosphorylation of histone $\mathrm{H} 3$ on serine 10 (H3deP) and deacetylation of $\mathrm{H} 4$ (H4deAc), which decreases the expression of host genes, B) interaction of InlB with its receptor Met targets SIRT2 to chromatin, where it deacetylates histone $\mathrm{H} 3$ on lysine 18 (H3K18deAc), which represses host genes transcription, and C) secreted LntA accumulates in the host nucleus, interacts with the chromatin-associated repressor BAHD1 and thereby promotes transcription of interferon-stimulated genes (ISG). 